\title{
Poster: Probabilistic Routing in Intermittently Connected Networks
}

\author{
Anders Lindgren \\ Luleå University of Technology \\ S-971 87 Luleå, Sweden \\ dugdale@sm.luth.se
}

\author{
Avri Doria \\ Luleå University of Technology \\ S-971 87 Luleå, Sweden \\ avri@acm.org
}

\author{
Olov Schelén \\ Luleå University of Technology \\ S-971 87 Luleå, Sweden \\ olov@sm.luth.se
}

\begin{abstract}
We consider the problem of routing in intermittently connected networks. In such networks there is no guarantee that a fully connected path between source and destination exist at any time, rendering traditional routing protocols unable to deliver messages between hosts. We propose a probabilistic routing protocol for such networks.
\end{abstract}

\section{Categories and Subject Descriptors}

C.2.1 [Computer-Communication Networks]: Network Architecture and Design

\section{General Terms}

Algorithms, Design

\section{Keywords}

Delay Tolerant Networks, routing, intermittently connected networks

\section{INTRODUCTION}

Normally, one of the most basic requirements for enabling two nodes to communicate through a network is that there exist a fully connected path between them. However, there are scenarios where this is not the case, but where it still would be desirable to allow communication between nodes. Such scenarios include communication between villages of the Saami population of reindeer herders in the north of Sweden [4], and other aboriginal populations and populations in poor regions [6], but also satellite communication [7], sensor networking [1], and other areas where the Delay Tolerant Networking (DTN) architecture [2] is of interest. In such networks, the mobility of nodes can be used to enable the delivery of messages - for example, even if there never is a path between nodes $\mathrm{A}$ and $\mathrm{C}, \mathrm{A}$ might meet node $\mathrm{B}$ and give a message to it, and $\mathrm{B}$ might later encounter $\mathrm{C}$, delivering the message to its final destination. In subsequent sections,

Permission to make digital or hard copies of all or part of this work for personal or classroom use is granted without fee provided that copies are not made or distributed for profit or commercial advantage and that copies bear this notice and the full citation on the first page. To copy otherwise, to republish, to post on servers or to redistribute to lists, requires prior specific permission and/or a fee.

MobiHoc'03, June 1-3, 2003, Annapolis, Maryland, USA.

Copyright 2003 ACM 1-58113-684-6/03/0006 ...\$5.00. some possible approaches for routing in a network where connectivity is intermittent are discussed, and a probabilistic routing protocol for such networks is outlined.

\section{RELATED WORK}

Vahdat and Becker present a protocol for epidemic routing in intermittently connected networks [8]. When two nodes encounter each other, they exchange messages being carried (subject to buffer space), thus causing the messages to spread through the network like an epidemic of a disease. This approach ensures that a message reaches its destination as soon as possible, but it also wastes a lot of resources through unnecessary message transfers.

Due to the ambiguity in deciding what the best next hop is in the networks discussed here, Chen and Murphy propose that applications should be able to affect that through the introduction of a utility function [3], allowing applications to specify weights of several factors influencing the function. In the proposed solution, called Disconnected Transitive Communication, a discovery protocol is used to find the best next hop within the cluster of currently connected nodes.

Grossglauser and Tse approach this kind of routing from a slightly different point of view [5]. One major problem with ad hoc networks is that due to interference of concurrent transmissions between nodes they scale badly. By only doing local communications between neighbors and instead relying on the movement of nodes to bring a message to its destination, it is shown that this problem can be mitigated.

\section{PROBABILISTIC ROUTING}

Most users usually do not move around completely randomly, and movement patterns are thus likely to be predictable, such that if a location has been frequently visited in the past, it is likely that it will be visited again in the future. We would like to make use of this observation to improve routing performance by doing probabilistic routing. A probabilistic metric called delivery predictability, is established at each node for each known destination indicating the predicted chance of that node delivering a message to that destination. When a node encounters another node, they exchange information about the delivery predictabilities they have and update their own information accordingly. Based on the delivery predictabilities, a decision is then made on whether or not to forward a certain message to this node.

\subsection{Delivery predictability calculation}

The protocol relies on the delivery predictability metric, $P \in[0,1]$, that should reflect the probability of encountering 
a certain node. That metric should be used to support the decision of whether or not to forward a message to a certain node.

Whenever a node is encountered, the metric should be updated according to (1), where $P_{(a, b)}$ is the delivery predictability node $a$ has for node $b$, and $P_{\text {init }} \in[0,1]$ is an initialization constant. This ensures that nodes that are often encountered have a high delivery predictability.

$$
P_{(a, b)}=P_{(a, b)_{o l d}}+\left(1-P_{(a, b)_{o l d}}\right) \times P_{\text {init }}
$$

If a pair of nodes do not encounter each other in a while, they are less likely to be good forwarders of messages to each other, thus the delivery predictability values must age, being reduced in the process. The aging equation is shown in (2), where $\gamma \in[0,1)$ is the aging constant, and $k$ is the number of time units that have elapsed since the last time the metric was aged. The time unit used can differ, and should be defined based on the application and the expected delays in the targeted network.

$$
P_{(a, b)}=P_{(a, b)_{o l d}} \times \gamma^{k}
$$

The delivery predictability also has a transitive property, that is based on the observation that if node A frequently encounters node $\mathrm{B}$, and node $\mathrm{B}$ frequently encounters node $\mathrm{C}$, then node $\mathrm{C}$ probably is a good node to forward messages destined for node A to. Equation 3 shows how this transitivity affects the delivery predictability, where $\beta \in[0,1]$ is a scaling constant that decides how large impact the transitivity should have on the delivery predictability.

$$
P_{(a, c)}=P_{(a, c)_{o l d}}+\left(1-P_{(a, c)_{o l d}}\right) \times P_{(a, b)} \times P_{(b, c)} \times \beta
$$

\subsection{Decision making}

In traditional routing protocols, choosing where to forward a message is usually a simple task; the message is sent to the neighbor with the lowest cost path to the destination (usually meaning least number of hops). Normally the message is also only sent to a single node since the reliability of paths is relatively high. However, in the settings we envision here, things are completely different. When a message arrives there might not be a path to the destination so the node have to keep the message for a while and each time it encounters another node it must make a decision if it should forward the message to that node or not. It might also be sensible to forward a message to multiple nodes to increase the probability that a message is really delivered to its destination.

Unfortunately, these decisions are not trivial to make. In some cases it might be reasonable to select a fixed threshold and only give a message to nodes that have a delivery predictability over that threshold for the destination of the message. On the other hand, when encountering a node with a low delivery predictability, it is not certain that a node with a higher metric will be encountered within reasonable time, thus there can also be situations where we might want to be less strict in deciding who to give messages to. Further, there is the problem of deciding how many nodes to give a certain message to. Distributing a message to a large number of nodes increases the probability of delivering the message to its destination, but more resources are be wasted. On the other hand, giving a message to only a few nodes (maybe just a single node) will use little resources, but the probability of delivering a message might be lower, and the incurred delay high. A number of trade-offs must be considered upon making the decision. Thus, we think the strategy adopted for a particular message should be chosen on a per-message basis to allow applications to cater for the special needs of their data. One situation that must be dealt with is the case where a system have just been started such that no delivery predictability information is available (or if mobility is such that prediction is difficult). In such a case, the protocol should fall back to some (possibly randomized) variant of epidemic routing.

\section{FUTURE WORK}

The use of acknowledgements to remove messages from the network after delivery to their destination will be investigated. Neglecting to do this will result in unnecessary transfers of messages, wasting valuable system resources. Extensive simulations of the proposed protocol will be run to evaluate the protocol, and to investigate the impact of different parameter settings and decision making strategies. Further, it would also be good to analytically evaluate the protocol to determine the suitability of the equations chosen for calculation of the delivery predictability.

\section{REFERENCES}

[1] G. W. Boehlert, D. P. Costa, D. E. Crocker, P. Green, T. O'Brien, S. Levitus, and B. J. L. Boeuf. Autonomous pinniped environmental samplers; using instrumented animals as oceanographic data collectors. Journal of Atmospheric and Oceanic Technology, 18(11):1882-1893, 2001. 18 (11).

[2] V. G. Cerf, S. C. Burleigh, A. J. Hooke, L. Torgerson, R. C. Durst, K. L. Scott, K. Fall, and H. S. Weiss. Delay-tolerant network architecture. Internet Draft draft-irtf-dtnrg-arch-02.txt, March 2003.

[3] X. Chen and A. L. Murphy. Enabling disconnected transitive communication in mobile ad hoc networks. In Proc. of Workshop on Principles of Mobile Computing, colocated with PODC'01, Newport, RI (USA), pages 21-27, aug 2001.

[4] A. Doria, M. Udén, and D. P. Pandey. Providing connectivity to the saami nomadic community. In Proceedings of the 2nd International Conference on Open Collaborative Design for Sustainable Innovation (dyd 02), Bangalore, India, Dec 2002.

[5] M. Grossglauser and D. Tse. Mobility increases the capacity of ad-hoc wireless networks. IEEE/ACM Transactions on Networking, 10(4), Aug 2002.

[6] A. Pentland, R. Fletcher, and A. A. Hasson. A road to universal broadband connectivity. In Proceedings of the 2nd International Conference on Open Collaborative Design for Sustainable Innovation (dyd 02), Bangalore, India, Dec 2002.

[7] G. E. Prescott, S. A. Smith, and K. Moe. Real-time information system technology challenges for NASA's earth science enterprise. In Proceedings of The 20th IEEE Real-Time Systems Symposium, Phoenix, Arizona, Dec 1999.

[8] A. Vahdat and D. Becker. Epidemic routing for partially connected ad hoc networks. Technical Report CS-200006, Duke University, April 2000. 